\title{
Higgs measurements and searches as a portal to physics beyond the Standard Model
}

\author{
Dario Buttazzo* \\ INFN Sezione di Pisa, Largo B. Pontecorvo 3, 56127 Pisa, Italy \\ E-mail: dario.buttazzo@pi.infn.it
}

\begin{abstract}
The measurement of Higgs properties poses significant constraints on physics beyond the Standard Model. These are compared with direct searches of additional scalar states of an extended Higgs sector. As a simple example, we consider a scalar singlet coupled to the other particles only through its mixing with the Higgs boson, which appears in several motivated extensions of the Standard Model. The prospects for the discovery of a generic singlet at the various stages of the LHC, as well as at future high-energy colliders, are studied, and the reach of direct searches is compared with the precision attainable with Higgs couplings measurements. The results are then applied to the NMSSM and Twin Higgs.
\end{abstract}

Sixth Annual Conference on Large Hadron Collider Physics (LHCP2018)

4-9 June 2018

Bologna, Italy

\footnotetext{
*Speaker.
} 


\section{Introduction}

After the discovery of the Higgs boson by the ATLAS and CMS experiments, our knowledge of the scalar sector of the Standard Model (SM) has improved significantly. The couplings of the Higgs to the other SM particles has been measured so far with a precision of about $10 \%$, and further improvement is expected as the LHC accumulates more data and proceeds towards its highluminosity phase. Moreover, differential measurements of the various cross-sections start to be possible, giving access to more information than the one encoded in integrated signal strengths. While all the properties of the Higgs boson appear SM-like so far, fundamental questions such as the origin of electroweak symmetry breaking and the naturalness of the Fermi scale remain open. It is then natural to ask the following questions: What are the implications of the measurements of the properties of the Higgs boson for models beyond the SM? How do these measurements compare with direct searches for new states? Is the boson observed at the LHC the only scalar particle, or are there other Higgs-like states around the Fermi scale?

Clearly, these questions cannot be answered in a fully model-independent way. A prime example where Higgs measurements play a dominant role is the one of composite Higgs models. Generically speaking, in this class of theories all operators compatible with the symmetries are generated at a given scale $f$ by a strong interaction. Deviations in Higgs couplings of the order of $v^{2} / f^{2}$ are expected, and their measurement is directly translated into a bound on the tuning of the electroweak scale. A different class of theories where large deviations in Higgs couplings can appear is constituted by weakly coupled models with an extended Higgs sector. Here the dominant effects in Higgs physics are generated at tree-level through the mixing among scalar states. Significative examples of this type are supersymmetric theories. In this case, Higgs properties are directly related to the mass of the extra scalar states, but the connection with the tuning of the electroweak scale is less immediate. As an example, in the Minimal Supersymmetric Standard Model (MSSM) the Higgs fit excludes a light second scalar doublet, but the value of the weak scale is determined by the squark masses which are independent from Higgs couplings deviations. In the NMSSM, on the other hand, where the tree-level value of the Higgs mass is modified, the relation between Higgs couplings, scalar masses, and tuning is very different, as can be seen in figure 1 .

In this contribution we will analyse the simplest possible example of extended Higgs sector: a real scalar singlet added to the SM. Despite its extreme simplicity, this physical situation arises as the low energy limit of several motivated theories: the NMSSM, Twin Higgs, models giving a first order electroweak phase transition, and models with a dark sector coupled to the SM through the Higgs portal. The phenomenology can be described by means of a small number of parameters essentially the mass of the new state $m_{\phi}$ and the mixing angle $\gamma$ - and can be used as a benchmark to compare the direct and indirect reach of the LHC and future colliders. Since the new state is a singlet, both Higgs couplings and direct searches are expected to be more powerful probes in generic models. For example, in models with a second Higgs doublet (such as the MSSM), Higgs measurements can probe a large fraction of the parameter space, and the remaining regions can usually be explored with direct searches for fermionic resonances ( $b b, t t, \tau \tau$, see figure 1$)$.

In the following, after briefly reviewing the main properties of a generic singlet-like scalar, I shall present the constraints on the existence of such a particle that arise from both direct searches and Higgs couplings precision measurements. 

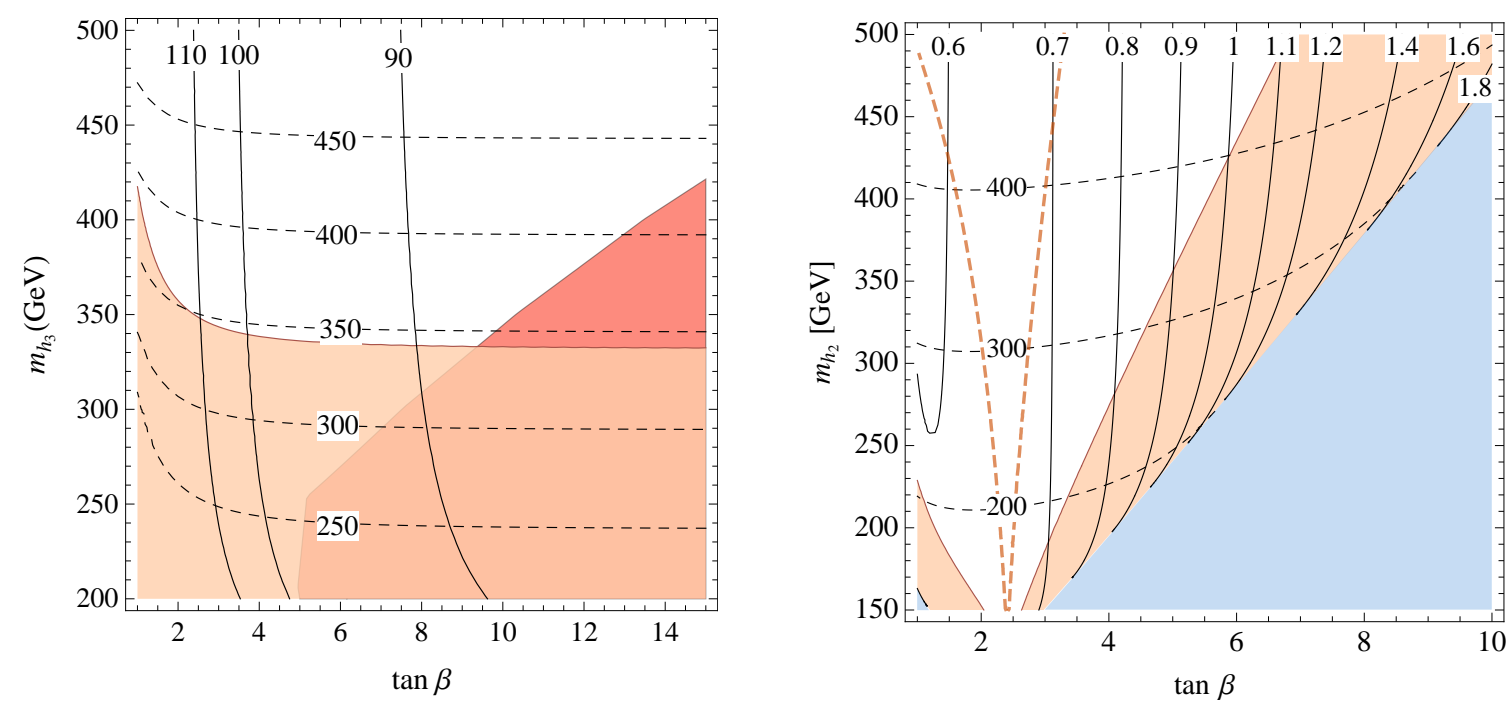

Figure 1: Left: exclusions in the plane ( $\left.\tan \beta, m_{H}\right)$ for the MSSM, from Higgs coupling measurements (pink) and direct searches in the $H \rightarrow \tau \tau$ channel (red). The black lines are the values of the one-loop contribution to $m_{h}$, the dashed lines show the mass of the charged Higgs. Right: the same exclusions in the heavy singlet limit of the NMSSM. The coloured dashed line is the $300 \mathrm{fb}^{-1}$ reach. Figures from [9].

\section{Scalar singlets}

Let us consider the following Lagrangian,

$$
\mathscr{L}=\mathscr{L}_{\mathrm{SM}}+\frac{1}{2}\left(\partial_{\mu} S\right)^{2}-\frac{m_{S}^{2}}{2} S^{2}-\frac{\lambda_{H S}}{2} S^{2}|H|^{2}-a_{H S} S|H|^{2}-V(S)
$$

where $S$ is a singlet under the SM gauge interactions. In general, for $a_{H S} \neq 0$, the neutral components in $H$ and $S$ will mix. Let us call $h$ and $\phi$ the two neutral, CP-even propagating degrees of freedom,

$$
h=h^{0} \cos \gamma+s^{0} \sin \gamma, \quad \phi=-h^{0} \sin \gamma+s^{0} \cos \gamma,
$$

with masses $m_{h}=125 \mathrm{GeV}$ and $m_{\phi}$, and mixing angle $\gamma$. As a consequence, both physical scalar states are coupled to SM particles, hence they can both be produced at colliders and be observed by means of their visible decays.

In a weakly interacting theory, the couplings of $h$ and $\phi$ are just the ones of a standard Higgs boson with the same mass, rescaled by a universal factor of $\cos \gamma$ or $\sin \gamma$, respectively. As a consequence, their signal strengths $\mu_{h, \phi}$ are

$$
\begin{aligned}
\mu_{h} & =\mu_{\mathrm{SM}}\left(m_{h}\right) \times c_{\gamma}^{2}, \\
\mu_{\phi \rightarrow V V, f f} & =\mu_{\mathrm{SM}}\left(m_{\phi}\right) \times s_{\gamma}^{2} \times\left(1-\mathrm{BR}_{\phi \rightarrow h h}\right), \\
\mu_{\phi \rightarrow h h} & =\sigma_{\mathrm{SM}}\left(m_{\phi}\right) \times s_{\gamma}^{2} \times \mathrm{BR}_{\phi \rightarrow h h},
\end{aligned}
$$

where $\mu_{\mathrm{SM}}(m)$ is the corresponding signal strength of a SM Higgs with mass $m$, and the branching ratio of $\phi$ into two $125 \mathrm{GeV}$ Higgs bosons, $\mathrm{BR}_{\phi \rightarrow h h}$, is a free quantity. The second state $\phi$ behaves 
like a heavy SM Higgs boson, with reduced couplings and an additional decay width into $h h$. The phenomenology of the Higgs system is therefore completely described by three parameters: $m_{\phi}$, $\sin \gamma$, and $\mathrm{BR}_{\phi \rightarrow h h}$. In the limit of large $m_{\phi}$, however, the Goldstone boson equivalence theorem sets the relations

$$
\mathrm{BR}_{\phi \rightarrow h h}=\mathrm{BR}_{\phi \rightarrow Z Z}=\frac{1}{2} \mathrm{BR}_{\phi \rightarrow W W} .
$$

Notice that the mixing angle $\gamma$ and $m_{\phi}$ are not independent quantities, since the former has to vanish when the mass tends to infinity. In a generic theory $\sin \gamma \approx m_{h} / m_{\phi}$, but if some additional symmetry suppresses $a_{H S}$ in (2.1) one can have also $\sin \gamma \approx m_{h}^{2} / m_{\phi}^{2}$. The exact formulae for the $h h h$ and $\phi h h$ couplings are reported in reference [1].

\subsection{Higgs couplings}

The measurement of the Higgs signal strengths provides a constraint on the mixing angle $\gamma$ through eq. (2.3). At present, a global fit to $8 \mathrm{TeV}$ LHC data constrain it to be $s_{\gamma}^{2}<0.13$ at $95 \%$ C.L. [2], and similar results can be obtained from the $13 \mathrm{TeV}$ data. Projections for the reach of future hadron and lepton colliders [3] are listed in Table 1.

Large modifications to the triple Higgs coupling can arise in some regions of the parameter space, even if the deviation in the signal strengths is moderate. Future collider experiments, and even the LHC, could in principle be sensitive to these modifications. More details about Higgs couplings can be found in [1].

\subsection{Direct searches}

The main decay channels of a heavy singlet are into a pair of $W$ and $Z$ vector bosons, or into a pair of Higgs bosons, if kinematically allowed.

Both the ATLAS and CMS collaborations provide a combined limit from all the $W W$ and $Z Z$ channels, with the strongest bound always coming from searches in the $4 \ell$ and $2 \ell 2 v$ final states [4]. In the di-Higgs channel, the main constraint comes from the $4 b$ final state [5]. All these searches are already sensitive to cross-sections smaller than the ones for a SM Higgs at the same mass, and exceed the reach of Higgs coupling measurements for low enough $m_{\phi}$.

Projections for future hadron colliders have been obtained in [1], rescaling the expected LHC limits with the parton luminosities of the backgrounds, following the procedure presented in [6]. The colliders that have been considered are: the $8 \mathrm{TeV}, 13 \mathrm{TeV}$, and $14 \mathrm{TeV}$ LHC, its highluminosity upgrade, a possible $33 \mathrm{TeV}$ energy upgrade, and a futuristic $100 \mathrm{TeV}$ FCC-hh. In [7] a

\begin{tabular}{|c|c|c|c|c|c|}
\hline$p p$ & LHC8 & LHC14 & HL-LHC & HE-LHC & FCC-hh \\
\hline$s_{\gamma}^{2}$ & 0.13 & $0.08-0.12$ & $0.04-0.08$ & $?$ & $?$ \\
$\left|\Delta g_{h h h} / g_{h h h}^{\text {SM }}\right|$ & - & 6 & 0.5 & 0.2 & 0.08 \\
\hline \hline$e^{+} e^{-}$ & ILC500 & ILC1000 & HL-ILC & CLIC & FCC-ee \\
\hline$s_{\gamma}^{2}$ & 0.02 & 0.02 & $4 \times 10^{-3}$ & $2-3 \times 10^{-3}$ & $10^{-3}$ \\
$\left|\Delta g_{h h h} / g_{h h h}^{\text {SM }}\right|$ & 0.83 & 0.46 & $0.1-0.2$ & $0.1-0.2$ & - \\
\hline
\end{tabular}

Table 1: Current and expected precisions on Higgs couplings [2, 3]. 


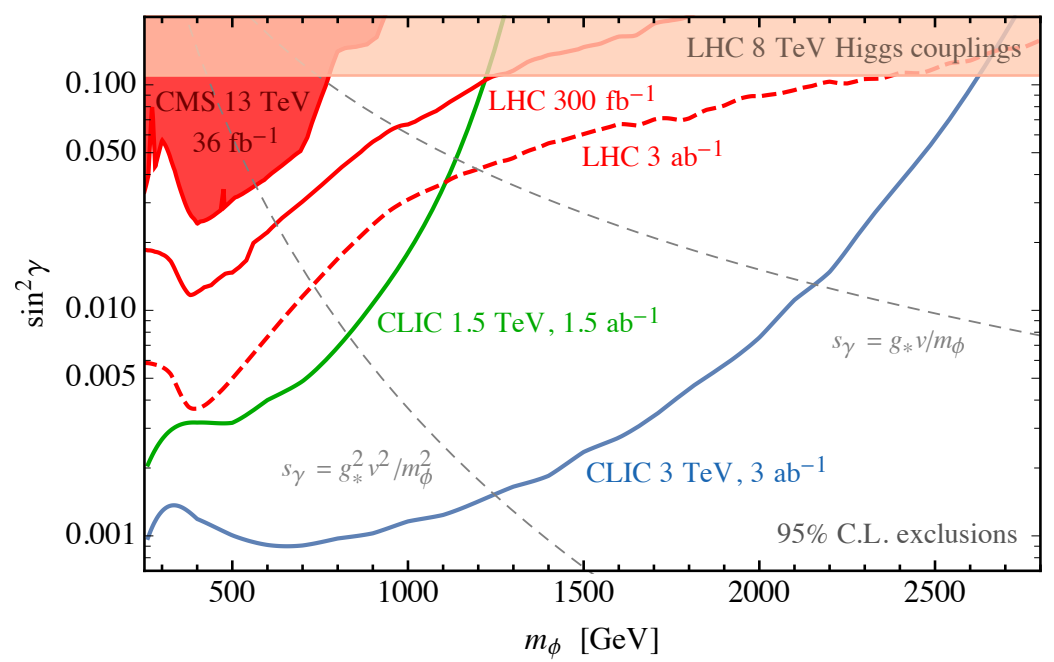

Figure 2: Comparison between the combined reach of direct searches and Higgs coupling measurements, in the plane $m_{\phi}-M_{h h}$. $\mathrm{BR}_{\phi \rightarrow h h}$ has been fixed to 0.25 for simplicity. The dashed lines show two examples of scaling of $\sin ^{2} \gamma$ with the mass $m_{\phi}$ (with $g_{*}=1$ ). Figure from [7].

study for high energy lepton colliders has been performed, obtaining the reach for scalar singlets at the $1.5 \mathrm{TeV}$ and $3 \mathrm{TeV}$ CLIC and at high-energy muon colliders with $6 \mathrm{TeV}$ and $14 \mathrm{TeV}$.

Figure 2 shows the present and future limits on the Higgs-singlet mixing angle, both from direct searches and Higgs couplings measurements. The direct exclusion at LHC (red) is dominated by $\phi \rightarrow V V$, while the reach at CLIC (blue and green) comes from $\phi \rightarrow h h$. We have fixed $\mathrm{BR}_{\phi \rightarrow h h}=1 / 4$ in the figure.

\section{Explicit models}

\subsection{Supersymmetry}

The Higgs sector of the NMSSM [8] contains the two doublets $H_{u, d}$, plus a singlet chiral superfield $S$, coupled through a Yukawa interaction $\lambda H_{u} H_{d} S$ in the superpotential. An extra contribution to the Higgs mass is generated at tree-level by $\lambda$, and reduces the size of the radiative correction needed to obtain $125 \mathrm{GeV}$. At the same time, the fine-tuning of the electroweak scale $v$ is reduced.

In the decoupling limit for the heavy doublet, the CP-even states are the SM Higgs and the singlet, and can be matched to the previous scenario [9]. The upper limit on the SM-like Higgs boson mass is

$$
m_{h}^{2} \leq m_{Z}^{2} c_{2 \beta}^{2}+v^{2} \lambda^{2} s_{2 \beta}^{2}+\Delta^{2},
$$

where $\Delta$ is the radiative correction and $\tan \beta=v_{u} / v_{d}$. Figure 3 (left) shows the current exclusions and projections from both direct searches and Higgs couplings, in the plane $m_{\phi}-\tan \beta$, for fixed values of $\lambda=1$ and $\Delta=80 \mathrm{GeV}$.

\subsection{Twin Higgs}

In Twin Higgs models [10], a naturally light Higgs is obtained without the presence of coloured particles close to the $\mathrm{TeV}$ scale. This is achieved introducing a copy of the SM field content and 

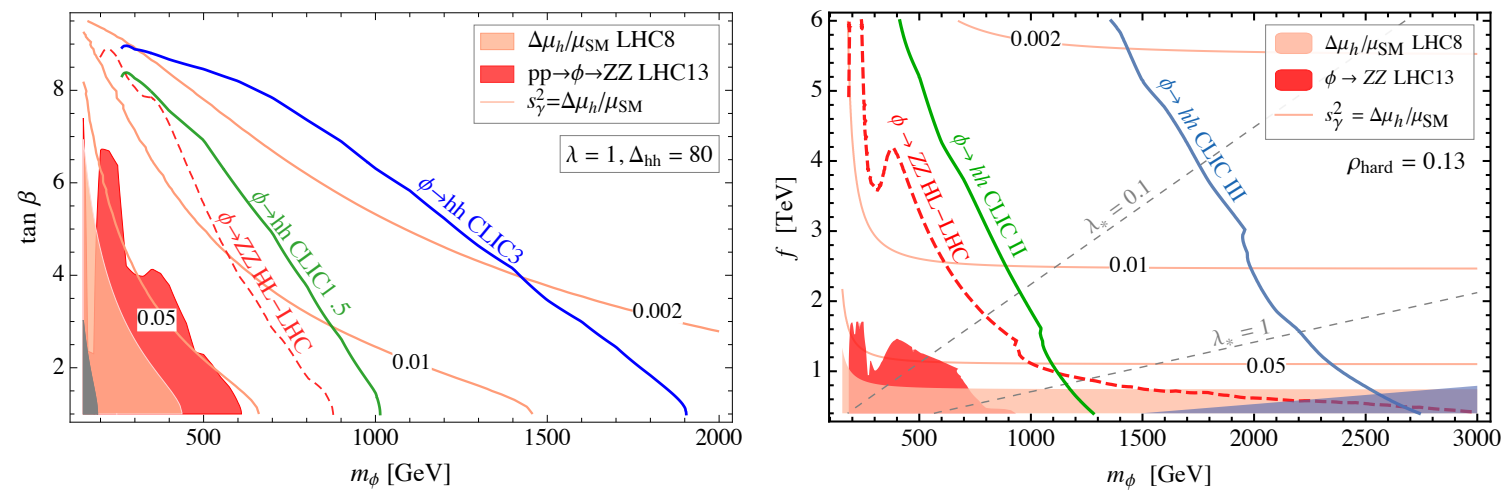

Figure 3: Current exclusions and projections for the NMSSM singlet with $\lambda=1$ and $\Delta=80 \mathrm{GeV}$ (left), and the Twin Higgs radial mode (right). The notation is the same as in Figure 2. In the purple region the width $\Gamma_{\phi}>m_{\phi}$. Figures from [7].

gauge symmetries, $\mathrm{SM}_{\mathrm{A}} \times \mathrm{SM}_{\mathrm{B}}$. The Higgs potential has an approximate global $\mathrm{SO}(8)$ symmetry, which is spontaneously broken at a scale $f$, and the Higgs $h=H_{\mathrm{A}} \cos \gamma+H_{\mathrm{B}} \sin \gamma$ is a Goldstone boson of this breaking. In presence of a $Z_{2}$ symmetry $\mathrm{A} \leftrightarrow \mathrm{B}$, quadratic "divergences" in the Higgs mass cancel between the A and B sectors, while all the new Twin particles are SM singlets.

The phenomenology of the "radial mode" $\phi=H_{\mathrm{B}} \cos \gamma-H_{\mathrm{A}} \sin \gamma$ is described by eq. (2.4), (2.5). The mixing angle is proportional to $v / f$,

$$
\sin ^{2} \gamma=\frac{v^{2}}{f^{2}}-\frac{m_{h}^{2}}{m_{\phi}^{2}-m_{h}^{2}}\left(1-2 \frac{v^{2}}{f^{2}}\right)+\frac{2 \rho v^{2}}{m_{\phi}^{2}-m_{h}^{2}}\left(1-\frac{v^{2}}{f^{2}}\right),
$$

where $\rho$ measures the amount of "hard" breaking of the $\mathrm{A} \leftrightarrow \mathrm{B}$ symmetry. The only difference with respect to the previous cases is the presence of an invisible width into $W_{\mathrm{B}}$ and $Z_{\mathrm{B}}$ bosons. Figure 3 (right) illustrates the present and future constraints in the plane $m_{\sigma^{-}} f$. One can see that direct searches for the radial mode are the most powerful probe for a Twin Higgs scenario, at least for not too large values of $m_{\sigma}$ and $f$.

\section{Conclusions}

Searches for new scalars at colliders can be an important probe for the extended Higgs sectors of many physically motivated models, and complementary to the measurement of Higgs couplings. Particularly simple, and at the same time of physical relevance, is the case of scalar singlets. As opposed to additional doublets, which decay preferentially into fermions, a heavy singlet decays dominantly into bosonic final states through the mixing with the SM Higgs. By means of only three parameters that determine the phenomenology in a completely general way, the reach of future colliders in the relevant $V V$ and $h h$ channels has been studied. The results have then been interpreted in two examples of motivated scenarios beyond the SM: the NMSSM and Twin Higgs. The main message is that already the second run of the LHC can efficiently probe the presence of additional scalar states, both directly and indirectly, and will provide valuable information about physics beyond the SM in the near future. 


\section{Acknowledgements}

I thank Diego Redigolo, Filippo Sala, and Andrea Tesi for the various collaborations on which the presented results are based. This research is supported by the INFN grant "FLAVOR".

\section{References}

[1] D. Buttazzo, F. Sala, A. Tesi, JHeP 1511 (2015) 033, [arXiv:1505.05488].

[2] ATLAS ANd CMS collaborations, JHEP 08 (2016) 045, [ARXiv: 1606.02266].

[3] S. Dawson et al., Working Group Report: Higgs boson, COMmunity Summer Study 2013: SNOWMASS ON THE MISSISSIPPI (2013), [ARXIV:1310.8361].

[4] CMS Collaboration, JHEP 1510 (2015) 144, [arXiv: 1504.00936].

[5] CMS collaboration, Phys. Lett. B 749 (2015) 560, [arXiv:1503.04114].

[6] A. THAMm, R. TORRE, A. Wulzer, JHEP 1507 (2015) 100, [ARXIV:1502.01701].

[7] D. Buttazzo, D. Redigolo, F. SAla And A. Tesi, ARXiv:1807.04743.

[8] U. Ellwanger, C. Hugonie, A. M. Teixeira, Phys. Rept. 496 (2010) 1, [ARXIV:0910.1785].

[9] R. BARBIERI et al., PHYS. Rev. D 87 (2013) 115018, [ARXIV:1304.3670].

[10] Z. Chacko, H.-S. Goh, R. Harnik, Phys. Rev. Lett. 96 (2006) 231802, [HeP-PH/0506256]. 